\title{
ANALYSIS OF NON-LINEAR TRANSMISSION LINES BY FREQUENCY DOMAIN PERTURBATION METHOD
}

\author{
AKIO USHIDA, YUICHI TANJI AND YOSHIFUMI NISHIO
}

Department of Electrical and Electronic Engineering, Faculty of Engineering, Tokushima University, Tokushima 770, Japan

\section{SUMMARY}

We discuss a numerical method for solving non-linear transmission lines in the frequency domain. Such transmission lines are widely used for communications such as in GaAs integrated circuits and varactor diode circuits. The circuit equations are described by non-linear partial differential equations, so their analysis is very complicated compared with that of linear transmission lines.

In this paper we propose a frequency-domain perturbation method for weakly non-linear transmission lines where the wave-forms are approximated by Fourier expansions and each frequency component is calculated by a modified perturbation method. To improve convergence, we introduce two new techniques, the compensation method and the homotopy method, which help to make the iteration stable and can be applied to a wide class of non-linear transmission lines. We have analysed shock wave phenomena in example. (C) 1997 by John Wiley \& Sons, Ltd. Int. J. Circ. Theor. Appl., vol. 25, 95-105 (1997)

(No. of Figures: 6; No. of Tables: 0; No. of Refs: 20.)

\section{INTRODUCTION}

Recently the analysis and design of high-speed non-linear systems have become more important in the fields of communications and LSI chips. On the other hand, fast-rise-time electrical devices are crucial for high-speed digital and analogue applications and for wide-bandwidth electronic systems. In GaAs non-linear transmission lines, ${ }^{1}$ varactor diode circuits and superconducting transmission lines ${ }^{2}$ the capacitors have non-linear voltagecharge characteristics. The velocity of a travelling wave in these non-linear transmission lines is given by a function of amplitudes as follows:

$$
\frac{\mathrm{d} x}{\mathrm{~d} t}=\frac{1}{\sqrt{ } L(i) C(v)}
$$

If the capacitor and/or inductor are decreasing functions of voltages and currents, the velocity will increase with their amplitudes. Therefore it has an interesting property that the higher part of the wave-form is faster than the lower part of it. This property is applied to many purposes such as picosecond pulse compression, broadband phase modulation, ${ }^{1}$ picosecond shock wave generation, ${ }^{3,4}$ MESFET gate mixers ${ }^{5}$ and so on. Since it is not very easy to analyse non-linear transmission lines, some papers discuss the phenomena from the physical point of view. ${ }^{1-3,6-8}$

On the other hand, the harmonic balance method is known as a powerful method for solving non-linear circuits when the wave-form does not contain many higher harmonics. In Reference 9, shock waves in nonlinear transmission lines are analysed by the harmonic balance method, where the line is approximated by a finite number of discrete lumped models driven by a sinusoidal wave generator. Note that the harmonic balance method can be efficiently applied only when the number of discrete models is not too large. The shock wave is one of the important phenomena in non-linear transmission lines. In Reference 10 it has been analysed by applying the difference approximation to the non-linear partial differential equations. In Reference 11, non-linear wave propagation phenomena are analysed by two-dimensional FFT. On the other hand, device models of IC chips are also described by non-linear partial differential equations ${ }^{12,13}$ and estimation of the 
delay time bound is also an important problem in the design of IC chips. In Reference 12 it is calculated using the $R C$ discrete model.

In this paper we discuss an efficient numerical method for solving non-linear transmission lines. We approximate the wave-form by a Fourier expansion at each point on the line and the coefficients are calculated by a perturbation technique. Although the perturbation method is only applicable to weakly non-linear systems, we have improved the property by introducing two techniques. The first one reduces the perturbed term by introducing a negative compensation component. The second one gradually increases the perturbed term step-by-step from zero to a specified value, which is the same technique as the homotopy method. ${ }^{14}$ The ideas will be applicable to the analysis of wide classes of non-linear circuits.

In Section 3 we apply our method to the analysis of shock waves in non-linear transmission lines. We find from the example that the convergence ratio is greatly improved by the introduction of the compensation and homotopy methods.

\section{FREQUENCY DOMAIN PERTURBATION METHOD}

\subsection{Perturbation method applied to non-linear transmission lines}

There are many kinds of non-linear elements such as Schottky barrier, metal-insulator-semiconductor (MIS) and GaAs integrated transmission lines ${ }^{1-4,9}$ which are used for harmonic and shock wave generation at microwave lengths. Their structures can be approximately described by a discrete model per small length $(\mathrm{d} x)$ in the spatial co-ordinate as shown in Figure 1. Thus the circuit is described by the non-linear partial differential equations

$$
-\frac{\partial v}{\partial x}=\frac{\partial \phi_{\mathrm{L}}}{\partial t}+v_{\mathrm{R}}, \quad-\frac{\partial i}{\partial x}=\frac{\partial q_{\mathrm{C}}}{\partial t}+i_{\mathrm{G}}
$$

where $\phi_{\mathrm{L}}$ and $q_{\mathrm{C}}$ are the flux of the non-linear inductor and the charge of the non-linear capacitor respectively. We assume the non-linear characteristics

$$
\begin{array}{ll}
i_{\mathrm{L}}=\Gamma \phi_{\mathrm{L}}+\varepsilon \hat{i}_{\mathrm{L}}\left(\phi_{\mathrm{L}}\right), & v_{\mathrm{R}}=R i_{\mathrm{R}}+\varepsilon \hat{v}_{\mathrm{R}}\left(i_{\mathrm{R}}\right) \\
v_{\mathrm{C}}=S q_{\mathrm{C}}+\varepsilon \hat{v}_{\mathrm{C}}\left(q_{\mathrm{C}}\right), & i_{\mathrm{G}}=G v_{\mathrm{G}}+\varepsilon \hat{i}_{\mathrm{G}}\left(v_{\mathrm{G}}\right)
\end{array}
$$

where $\varepsilon$ is a small constant and $\hat{i}_{\mathrm{L}}\left(\phi_{\mathrm{L}}\right), \hat{v}_{\mathrm{R}}\left(i_{\mathrm{R}}\right), \hat{v}_{\mathrm{C}}\left(q_{\mathrm{C}}\right)$ and $\hat{i}_{\mathrm{G}}\left(v_{\mathrm{G}}\right)$ are non-linear terms. From the circuit model in Figure 1 , we have the relations $i \equiv i_{\mathrm{L}}=i_{\mathrm{R}}$ and $v \equiv v_{\mathrm{C}}=v_{\mathrm{G}}$.

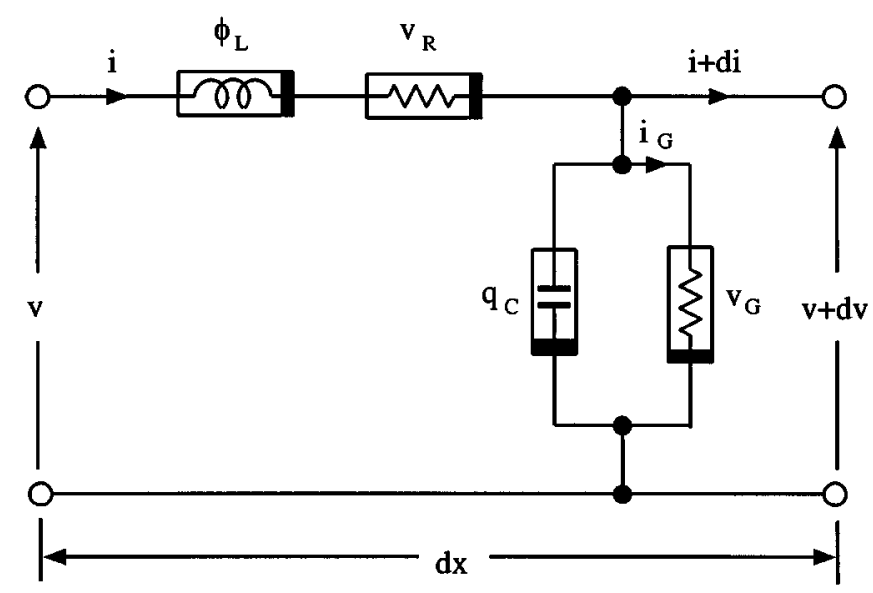

Fig. 1. Discrete model of non-linear transmission line 
To analyse shock waves, consider the input impulse wave-form

$$
e(t)= \begin{cases}E_{\mathrm{m}} \sin [(2 k \pi / T) t], & 0 \leqslant t \leqslant T / 2 k \\ 0, & T / 2 k \leqslant t \leqslant T\end{cases}
$$

where $T$ is the period of the impulse and $k$ is an integer. An example of this wave-form is shown in Figure 5 where $T=1 \mathrm{~ns}, E_{\mathrm{m}}=0 \cdot 25 \mathrm{~V}$ and $k=16$ (see Section 3 ).

In order to apply the frequency domain approach, we describe it by a Fourier expansion in the complex domain as

$$
e(t)=\sum_{k=-M}^{M} E_{k} \mathrm{e}^{\mathrm{j} k \omega t}, \quad \omega=2 \pi / T
$$

for sufficiently large $M$. Let us describe the steady-state responses in Fourier expansions as

$$
\begin{aligned}
v(x, t) & =\sum_{k=-M}^{M} V_{k}(x) \mathrm{e}^{\mathrm{j} k \omega t}, & i(x, t) & =\sum_{k=-M}^{M} I_{k}(x) \mathrm{e}^{\mathrm{j} k \omega t} \\
\phi_{\mathrm{L}}(x, t) & =\sum_{k=-M}^{M} \Phi_{k}(x) \mathrm{e}^{\mathrm{j} k \omega t}, & q_{\mathrm{C}}(x, t) & =\sum_{k=-M}^{M} Q_{k}(x) \mathrm{e}^{\mathrm{j} k \omega t}
\end{aligned}
$$

Substituting (5) into (1) and (2), we get the relations

$$
\begin{aligned}
-\frac{\mathrm{d} V_{k}}{\mathrm{~d} x} & =\mathrm{j} k \omega \Phi_{k}+R I_{k}+\varepsilon \hat{V}_{\mathrm{R}, k}(i) \\
-\frac{\mathrm{d} I_{k}}{\mathrm{~d} x} & =\mathrm{j} k \omega Q_{k}+G V_{k}+\varepsilon \hat{I}_{\mathrm{G}, k}(v) \\
I_{k} & =\Gamma \Phi_{k}+\varepsilon \hat{I}_{\mathrm{L}, k}\left(\phi_{\mathrm{L}}\right) \\
V_{k} & =S Q_{k}+\varepsilon \hat{V}_{\mathrm{C}, k}\left(q_{\mathrm{C}}\right)
\end{aligned}
$$

where $\varepsilon \hat{V}_{\mathrm{R}, k}(i), \varepsilon \hat{I}_{\mathrm{G}, k}(v), \varepsilon \hat{I}_{\mathrm{L}, k}\left(\phi_{\mathrm{L}}\right)$ and $\varepsilon \hat{V}_{\mathrm{C}, k}\left(q_{\mathrm{C}}\right)$ are the $k$ th frequency components obtained from the nonlinear terms. Observe that since the non-linear terms are functions of $2 M+1$ Fourier coefficients, the solutions of (6) satisfying the terminal relations are calculated by solving the non-linear two-point boundary value problem in $4(2 M+1)$ dimensions. ${ }^{15}$ This is really time-consuming when the frequency components are increased. Fortunately, if the non-linear terms are small enough compared with the linear terms, the perturbation method ${ }^{16}$ can be efficiently applied to solve (6).

Now let us assume the solutions at the $m$ th iteration as

$$
\begin{aligned}
v^{m}(x, t) & =\sum_{k=-M}^{M} V_{k}^{m}(x) \mathrm{e}^{\mathrm{j} k \omega t}, & i^{m}(x, t) & =\sum_{k=-M}^{M} I_{k}^{m}(x) \mathrm{e}^{\mathrm{j} k \omega t} \\
\phi_{\mathrm{L}}^{m}(x, t) & =\sum_{k=-M}^{M} \Phi_{k}^{m}(x) \mathrm{e}^{\mathrm{j} k \omega t}, & q_{\mathrm{C}}^{m}(x, t) & =\sum_{k=-M}^{M} Q_{k}^{m}(x) \mathrm{e}^{\mathrm{j} k \omega t}
\end{aligned}
$$

Then from (6) and (7) the perturbed equations are written in the forms

$$
\begin{aligned}
\frac{\mathrm{d} V_{k}^{m}}{d x} & =-(R+\mathrm{j} k \omega L) I_{k}^{m}-\varepsilon\left[\hat{V}_{\mathrm{R}, k}\left(i^{m-1}\right)-\mathrm{j} k \omega L \hat{I}_{\mathrm{L}, k}\left(\phi_{\mathrm{L}}^{m-1}\right)\right] \\
\frac{\mathrm{d} I_{k}^{m}}{\mathrm{~d} x} & =-(G+\mathrm{j} k \omega C) V_{k}^{m}-\varepsilon\left[\hat{I}_{\mathrm{G}, k}\left(v^{m-1}\right)-\mathrm{j} k \omega C \hat{V}_{\mathrm{C}, k}\left(q_{\mathrm{C}}^{m-1}\right)\right]
\end{aligned}
$$

where $L=1 / \Gamma, C=1 / S$ and

$$
\begin{aligned}
& \Phi_{k}^{m}=L\left[I_{k}^{m}-\varepsilon \hat{I}_{\mathrm{L}, k}\left(\phi_{\mathrm{L}}^{m-1}\right)\right] \\
& Q_{k}^{m}=C\left[V_{k}^{m}-\varepsilon \hat{V}_{\mathrm{C}, k}\left(q_{\mathrm{C}}^{m-1}\right)\right]
\end{aligned}
$$


Observe that relations (8a) and (8b) are ordinary differential equations w.r.t. the distance $x$ whose second terms on the right-hand side correspond to the forced terms obtained at the $(m-1)$ th iteration. $\Phi_{k}^{m}$ and $Q_{k}^{m}$ can be decided from (8c) and (8d) after solving (8a) and (8b).

The solutions of (8a) and (8b) are written as

$$
\begin{aligned}
& V_{k}^{m}(x)=A_{k}^{m} \mathrm{e}^{\lambda_{k} x}+B_{k}^{m} \mathrm{e}^{-\lambda_{k} x}+\varepsilon \hat{V}_{k}^{m}(x) \\
& I_{k}^{m}(x)=\frac{1}{Z_{k, 0}}\left(-A_{k}^{m} \mathrm{e}^{\lambda_{k} x}+B_{k}^{m} \mathrm{e}^{-\lambda_{k} x}\right)+\varepsilon \hat{I}_{k}^{m}(x)
\end{aligned}
$$

where the propagation constant $\lambda_{k}$ and characteristic impedance $Z_{k, 0}$ for the $k$ th frequency component are given by

$$
\lambda_{k}=\sqrt{ }[(\mathrm{j} k \omega L+R)(\mathrm{j} k \omega C+G)], \quad Z_{k, 0}=\sqrt{ }\left(\begin{array}{c}
\mathrm{j} k \omega L+R \\
\mathrm{j} k \omega C+G
\end{array}\right)
$$

The first two terms of (9) correspond to the zero-input responses; $\varepsilon \hat{V}_{k}^{m}(x)$ and $\varepsilon \hat{I}_{k}^{m}(x)$ are the zero-state responses ${ }^{17}$ which can be calculated by a numerical intergration technique such as backward difference. ${ }^{18}$ Here the constant parameters $A_{k}$ and $B_{k}$ in (9) are evaluated by the boundary conditions of the transmission line. When it is terminated by the impedance $Z_{\mathrm{L}}$ at the far end, they are given as

$$
\begin{gathered}
A_{k}^{m}=\frac{\varepsilon\left[\hat{I}_{k}^{m}(l) Z_{\mathrm{L}}(\mathrm{j} k \omega)-\hat{V}_{k}^{m}(l)\right] Z_{k, 0}-\left[Z_{k, 0}-Z_{\mathrm{L}}(\mathrm{j} k \omega)\right] E_{k} \mathrm{e}^{-\lambda_{k} l}}{\left[Z_{k, 0}+Z_{\mathrm{L}}(\mathrm{j} k \omega)\right] \mathrm{e}^{\lambda_{k} l}-\left[Z_{k, 0}-Z_{\mathrm{L}}(\mathrm{j} k \omega)\right] \mathrm{e}^{-\lambda_{k} l}} \\
B_{k}^{m}=\frac{\varepsilon\left[-\hat{I}_{k}^{m}(l) Z_{\mathrm{L}}(\mathrm{j} k \omega)+\hat{V}_{k}^{m}(l)\right] Z_{k, 0}+\left[Z_{k, 0}+Z_{\mathrm{L}}(\mathrm{j} k \omega)\right] E_{k} \mathrm{e}^{\lambda_{k} l}}{\left[Z_{k, 0}+Z_{\mathrm{L}}(\mathrm{j} k \omega)\right] \mathrm{e}^{\lambda_{k} l}-\left[Z_{k, 0}-Z_{\mathrm{L}}(\mathrm{j} k \omega)\right] \mathrm{e}^{-\lambda_{k} l}}
\end{gathered}
$$

Thus the steady state wave-forms at the $m$ th iteration are estimated by $(5 a)$ and $(5 b)$.

The iteration will be continued until the variation

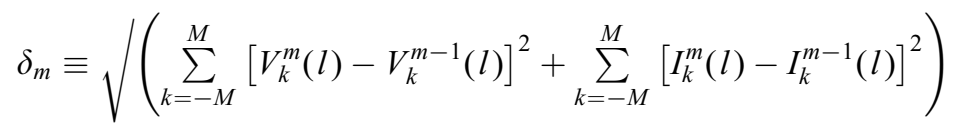

becomes sufficiently small for a given constant $\delta$. Note that we cannot say whether the solution wave-form is sufficiently accurate or not. Thus let us define the following residual error. Assume that the solutions for $M$ frequency components are $v_{M}(l, t)$ and $i_{M}(l, t)$ and that the exact solutions are $\hat{v}(l, t)$ and $\hat{i}(l, t)$. Then the residual error is defined as

$$
\varepsilon_{M} \equiv \sqrt{ }\left(\frac{1}{T} \int_{0}^{T}\left\{\left[v_{M}(l, t)-\hat{v}(l, t)\right]^{2}+\left[i_{M}(l, t)-\hat{i}(l, t)\right]^{2}\right\} \mathrm{d} t\right)
$$

Since it is impossible to get the exact solutions, we assume $v_{M^{\prime}}(x, t)$ and $i_{M^{\prime}}(x, t)$ as the exact solutions which are obtained with a larger number of frequency components $\left(M^{\prime} \gg M\right)$. Then we have

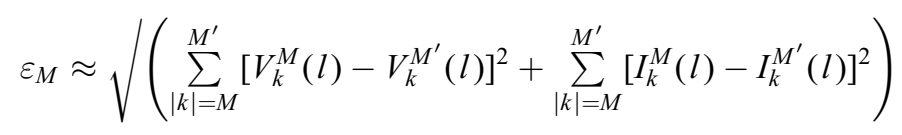

If the residual error $\varepsilon_{M}$ is not small enough, we need to choose a much larger $M$ for the approximation and again try our perturbation method.

\subsection{Improvements in perturbation method}

It is known that the perturbation method ${ }^{16}$ can be applied only to weakly non-linear circuits. In this subsection we improve the method such that it can be applied to a wider class of non-linear circuits.

Assume that the non-linear characteristics given in (2) are monotone increasing functions. For simplicity we describe the situation as

$$
u_{1}=H u_{2}+\varepsilon \hat{h}\left(u_{2}\right)
$$


where $u_{1}=\left(i_{\mathrm{L}}, v_{\mathrm{R}}, v_{\mathrm{C}}, i_{\mathrm{G}}\right)^{\mathrm{T}}, \quad u_{2}=\left(\phi_{\mathrm{L}}, i_{\mathrm{R}}, q_{\mathrm{C}}, v_{\mathrm{G}}\right)^{\mathrm{T}}$ and

$$
H=\operatorname{diag}(\Gamma, R, S, G), \quad \varepsilon \hat{h}\left(u_{2}\right)=\varepsilon\left(\hat{i}_{\mathrm{L}}\left(\phi_{\mathrm{L}}\right), \hat{v}_{\mathrm{R}}\left(i_{\mathrm{R}}\right), \hat{v}_{\mathrm{C}}\left(q_{\mathrm{C}}\right), \hat{i}_{\mathrm{G}}\left(v_{\mathrm{G}}\right)\right)^{\mathrm{T}}
$$

To improve the convergence ratio of our perturbation method, we try to reduce the perturbed term $\varepsilon \hat{h}\left(u_{2}\right)$. Let us introduce a small constant

$$
\Delta H \equiv \operatorname{diag}(\Delta \Gamma, \Delta R, \Delta S, \Delta G)
$$

as the compensation parameter in the form

$$
u_{1}=(H+\Delta H) u_{2}+\left[\varepsilon \hat{h}\left(u_{2}\right)-\Delta H u_{2}\right]
$$

The second term corresponds to the perturbed term, which can be greatly reduced by choosing a suitable $\Delta H$. We found from our numerical example that the convergence ratio is greatly improved even for a small compensation parameter $\Delta H$.

The homotopy method ${ }^{14}$ is sometimes used when the iteration method does not guarantee convergence. It has the property of global convergence. We will apply it to our perturbation method. Let us introduce a parameter $\rho$ for the non-linear elements as follows:

$$
u_{1}=(H+\Delta H) u_{2}+\rho\left[\varepsilon \hat{h}\left(u_{2}\right)-\Delta H u_{2}\right], \quad \rho: 0 \longrightarrow 1
$$

It is clear that relation (15) for $\rho=0$ corresponds to the linear case and that the non-linearity is gradually increased by choosing $\{\rho: 0 \rightarrow 1\}$. At $\rho=1$ it is reduced to the original equation. If we choose a small variation $\Delta \rho$ per iteration such as

$$
\rho^{m}=\rho^{m-1}+\Delta \rho
$$

our perturbation method can get the solution stably.

\subsection{Convergence conditions}

Now let us consider the convergence conditions of our perturbation method. For simplicity let us introduce the following notation for the variables in (8) and (9).

(a) For the solutions we set

$$
X_{1, k}^{m}(x)=\left(\begin{array}{c}
V_{k}^{m}(x) \\
I_{k}^{m}(x)
\end{array}\right), \quad X_{2, k}^{m}(x)=\left(\begin{array}{c}
\Phi_{k}^{m}(x) \\
Q_{k}^{m}(x)
\end{array}\right)
$$

(b) For the perturbed terms we set

$$
\hat{X}_{1, k}\left(w_{1}^{m-1}, x\right)=\left(\begin{array}{c}
\hat{V}_{\mathrm{R}, k}\left(i^{m-1}\right) \\
\hat{I}_{\mathrm{G}, k}\left(v^{m-1}\right)
\end{array}\right), \quad \hat{X}_{2, k}\left(w_{2}^{m-1}, x\right)=\left(\begin{array}{c}
\hat{I}_{\mathrm{L}, k}\left(\phi_{\mathrm{L}}^{m-1}\right) \\
\hat{V}_{\mathrm{C}, k}\left(q_{\mathrm{C}}^{m-1}\right)
\end{array}\right)
$$

where $w_{1}=(v, i)^{\mathrm{T}}$ and $w_{2}=\left(\phi_{\mathrm{L}}, q_{\mathrm{C}}\right)^{\mathrm{T}}$.

We describe the coefficient matrices of (8) as

$$
\begin{aligned}
A_{k} & =\left(\begin{array}{cc}
0 & R+\mathrm{j} k \omega L \\
G+\mathrm{j} k \omega C & 0
\end{array}\right), \\
C_{0} & =\left(\begin{array}{ll}
0 & L \\
C & 0
\end{array}\right)
\end{aligned}
$$

Now we describe the zero-state response of (9) in the integrations. Then we have from (9) and (10)

$$
\begin{aligned}
X_{1, k}^{m}(x)= & C_{1, k}(x) E_{k}+C_{2, k}(x) X_{1, k}^{m}(l)-\varepsilon \int_{0}^{x} \mathrm{e}^{-A_{k}(x-s)} \hat{X}_{1, k}\left(w_{1}^{m-1}, s\right) \mathrm{d} s \\
& -\varepsilon B_{k} \int_{0}^{x} \mathrm{e}^{-A_{k}(x-s)} \hat{X}_{2, k}\left(w_{2}^{m-1}, s\right) \mathrm{d} s \\
X_{2, k}^{m}(x)= & C_{0} X_{1, k}^{m}(x)-\varepsilon C_{0} \hat{X}_{2, k}\left(w_{2}^{m-1}, x\right)
\end{aligned}
$$

where $C_{1, k}(x)$ and $C_{2, k}(x)$ are obtained from (9) and (10). 
Let us adopt the norm ${ }^{19}$

$$
\|w(t)\| \equiv \sqrt{ }\left(\frac{1}{T} \int_{0}^{T} w^{2}(t) \mathrm{d} t\right)
$$

Assume that $w(t)$ is a periodic function of the period $T$ as follows:

$$
w(t)=\sum_{k=-M}^{M} W_{k} \mathrm{e}^{\mathrm{j} k \omega t}
$$

Then we have the Euclidean norm

$$
\|W\| \equiv \sqrt{ }\left(\left|W_{-M}\right|^{2}+\left|W_{-M+1}\right|^{2}+\cdots+\left|W_{M}\right|^{2}\right)
$$

Thus the two norms coincide with each other, i.e.

$$
\|w(t)\|=\|W\|
$$

Now we have the following convergence conditions.

\section{Theorem 1.}

Assume that for an approximate solution $\left(w_{1}^{0}, w_{2}^{0}\right)^{\mathrm{T}}$ there are constants $D_{1}, D_{2}, K_{0}, L_{1}$ and $L_{2}$ satisfying the following:

(i) Define the solution domain by

$$
\Omega \equiv\left\{w_{1}, w_{2} \mid\left\|w_{1}-w_{1}^{0}\right\| \leqslant D_{1},\left\|w_{2}-w_{2}^{0}\right\| \leqslant D_{2}\right\}
$$

(ii) Define the norms of the coefficient matrices by

$$
K_{0} \equiv\left\|C_{0}\right\|, \quad K_{1} \equiv \max _{-M \leqslant k \leqslant M} \frac{\left\|A_{k}^{-1}\right\| L_{1} l}{1-C_{2 k}}, \quad K_{2} \equiv \max _{-M \leqslant k \leqslant M} \frac{\left\|B_{k} A_{k}^{-1}\right\| L_{2} l}{1-C_{2 k}}
$$

where

$$
C_{2 k} \equiv \max _{0 \leqslant x \leqslant l}\left\|C_{2, k}(x)\right\|
$$

(iii) Assume that the perturbed terms satisfy the Lipschitz conditions:

$$
\begin{aligned}
& \left\|\hat{X}_{1}\left(w_{1}{ }^{\prime}, x\right)-\hat{X}_{1}\left(w_{1}{ }^{\prime \prime}, x\right)\right\| \leqslant L_{1}\left\|w_{1}{ }^{\prime}-w_{1}{ }^{\prime \prime}\right\| \\
& \left\|\hat{X}_{2}\left(w_{2}{ }^{\prime}, x\right)-\hat{X}_{2}\left(w_{2}{ }^{\prime \prime}, x\right)\right\| \leqslant L_{2}\left\|w_{2}{ }^{\prime}-w_{2}{ }^{\prime \prime}\right\|
\end{aligned}
$$

in $\Omega$ for all $0 \leqslant x \leqslant l$, where $L_{1}$ and $L_{2}$ are Lipschitz constants.

(iv) Set the maximum values of the variables in $x=[0, l]$ as

$$
X_{1, k}^{m}=\max _{0 \leqslant x \leqslant l}\left\|X_{1, k}^{m}(x)\right\|, \quad X_{2, k}^{m}=\max _{0 \leqslant x \leqslant l}\left\|X_{2, k}^{m}(x)\right\|
$$

Furthermore, if it satisfies the condition

$$
\kappa \equiv \varepsilon\|P\|<1, \quad \text { for } P=\left(\begin{array}{cc}
K_{1} & K_{2} \\
K_{0} K_{1} & K_{0}\left(K_{2}+L_{2}\right)
\end{array}\right)
$$

our perturbation method (18) will converge to the unique solution $\left(w_{1}, w_{2}\right)^{\mathrm{T}}$.

Proof. For non-linear transmission lines we can assume $\left\|\mathrm{e}^{-A_{k} x}\right\| \leqslant 1$ for $0 \leqslant x \leqslant l$. Hence we have from (18a) and $(21 b)^{17}$

$$
\left\|X_{1, k}^{m}(x)-X_{1, k}^{m-1}(x)\right\| \leqslant C_{2 k}\left\|X_{1, k}^{m}(l)-X_{1, k}^{m-1}(l)\right\|
$$




$$
\begin{aligned}
& +\varepsilon\left\|A_{k}^{-1}\right\| \int_{0}^{x}\left\|\hat{X}_{1, k}\left(w_{1}^{m-1}, s\right)-\hat{X}_{1, k}\left(w_{1}^{m-2}, s\right)\right\| \mathrm{d} s \\
& +\varepsilon\left\|B_{k} A_{k}^{-1}\right\| \int_{0}^{x}\left\|\hat{X}_{2, k}\left(w_{2}^{m-1}, s\right)-\hat{X}_{2, k}\left(w_{2}^{m-2}, s\right)\right\| \mathrm{d} s
\end{aligned}
$$

Applying the Lipschitz conditions (21c) and (21d), we have

$$
\sum_{k=-M}^{M}\left\|X_{1, k}^{m}-X_{1, k}^{m-1}\right\| \leqslant \varepsilon K_{1} \sum_{k=-M}^{M}\left\|X_{1, k}^{m-1}-X_{1, k}^{m-2}\right\|+\varepsilon K_{2} \sum_{k=-M}^{M}\left\|X_{2, k}^{m-1}-X_{2, k}^{m-2}\right\|
$$

Thus we have from (20)

$$
\left\|X_{1}^{m}-X_{1}^{m-1}\right\| \leqslant \varepsilon K_{1}\left\|X_{1}^{m-1}-X_{1}^{m-2}\right\|+\varepsilon K_{2}\left\|X_{2}^{m-1}-X_{2}^{m-2}\right\|
$$

In the same manner we have from $(18 \mathrm{~b})$

$$
\left\|X_{2}^{m}-X_{2}^{m-1}\right\| \leqslant K_{0}\left\|X_{1}^{m}-X_{1}^{m-1}\right\|+\varepsilon K_{0} L_{2}\left\|X_{2}^{m-1}-X_{2}^{m-2}\right\|
$$

Set $X=\left(X_{1}, X_{2}\right)^{\mathrm{T}}$. Then we have from (22) and (23)

$$
\left\|X^{m}-X^{m-1}\right\| \leqslant \kappa\left\|X^{m-1}-X^{m-2}\right\|
$$

Hence

$$
\left\|X^{m}-X^{m-1}\right\| \leqslant \kappa^{m-1}\left\|X^{1}-X^{0}\right\|
$$

Thus our perturbation method will converge to the solution $\bar{X}$ and the error bound of the approximate solution $X^{0}$ is estimated as

$$
\begin{aligned}
\left\|X^{m}-X^{0}\right\| & \leqslant\left\|X^{m}-X^{m-1}\right\|+\left\|X^{m-1}-X^{m-2}\right\|+\cdots+\left\|X^{1}-X^{0}\right\| \\
& \leqslant\left(\kappa^{m-1}+\kappa^{m-2}+\cdots+\kappa+1\right)\left\|X^{1}-X^{0}\right\| \\
& =\frac{1-\kappa^{m}}{1-\kappa}\left\|X^{1}-X^{0}\right\|
\end{aligned}
$$

Thus we have proved the convergence condition.

Q.E.D

We found from the Theorem 1 that our perturbation method will converge to an approximate solution $\bar{X}$ if the non-linear term $\varepsilon$ is sufficiently small.

\section{ILLUSTRATIVE EXAMPLE}

As an example of non-linear transmission lines, let us calculate the shock wave due to an impulse. Assume that the line is terminated by a resistor $R_{\mathrm{L}}=10 \Omega$. For simplicity, we assume that the inductor is linear and the other characteristics in (2) are given as

$$
i_{\mathrm{L}}=350 \times 10^{11} \phi_{\mathrm{L}}, \quad v_{\mathrm{R}}=0 \cdot 015 i_{\mathrm{R}}+0 \cdot 1 i_{\mathrm{R}}^{3}, \quad v_{\mathrm{C}}=10^{11} q_{\mathrm{C}}+0 \cdot 5 \times 10^{34} q_{\mathrm{C}}^{3}, \quad i_{\mathrm{G}}=0 \cdot 015 v_{\mathrm{G}}+0 \cdot 1 v_{\mathrm{G}}^{3}
$$




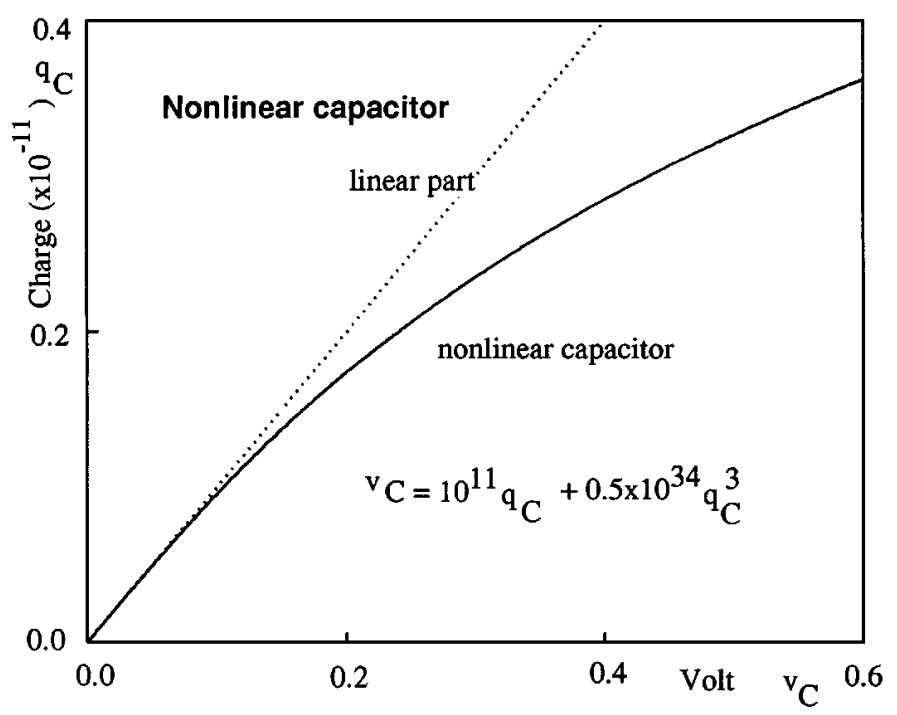

Fig. 2. Characteristic curve of non-linear capacitor: $v_{\mathrm{C}}=10^{11} q_{\mathrm{C}}+0 \cdot 5 \times 10^{34} q_{\mathrm{C}}^{3}$

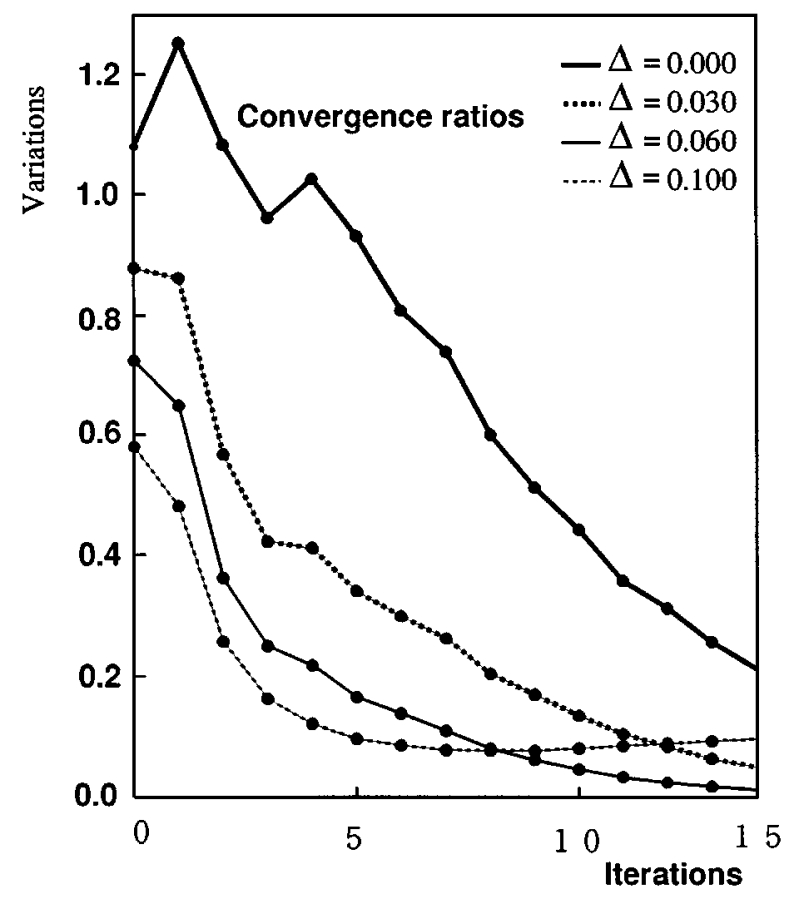

Fig. 3. Convergence ratios for various compensation parameters, where variations are defined by $\delta_{m}=\left\|X^{m}(l)-X^{m-1}(l)\right\|$

The $q-v$ characteristic curve is shown in Figure 2. We assume $E_{m}=0.25 \mathrm{~V}, T=1 \mathrm{~ns}$ and $k=16$ for the input impulse in (3). For the calculation of zero-state responses in (8) we applied the first- and/or second-order backward difference formulae ${ }^{18}$, where the step size is chosen as $\Delta x=l / 60$. 


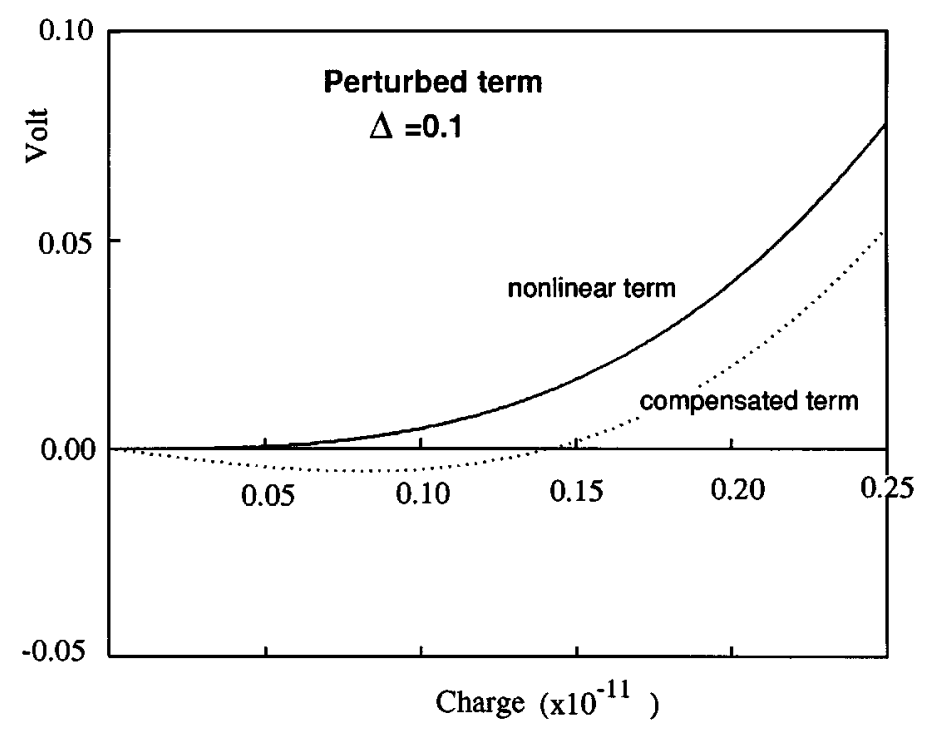

Fig. 4. Perturbed term by compensation method for $\Delta=0 \cdot 1 . \hat{v}_{\mathrm{C}}=0 \cdot 5 \times 10^{34} q_{\mathrm{C}}^{3}-0 \cdot 1 \times 10^{11} q_{\mathrm{C}}$

The convergence ratios are shown in Figure 3 for various compensation parameters $\Delta H$. In this case the variations are estimated by

$$
\delta_{m}=\left\|\left(\sum_{k=-128}^{128}\left[V_{k}^{m}(l)-V_{k}^{m-1}(l)\right]^{2}+\sum_{k=-128}^{128}\left[I_{k}^{m}(l)-I_{k}^{m-1}(l)\right]^{2}\right)^{1 / 2}\right\|
$$

We found from the results that the convergence will be greatly improved even for a small $\Delta$ in (14). Figure 4 shows the perturbed term for $\Delta=0 \cdot 1$ :

$$
\hat{v}_{\mathrm{C}}=0 \cdot 5 \times 10^{34} q_{\mathrm{C}}^{3}-0 \cdot 1 \times 10^{11} q_{\mathrm{C}}
$$

When we applied both the compensation method with $\Delta=0.03$ and the homotopy method with

$$
\rho^{m}=\rho^{m-1}+0 \cdot 1, \quad \text { for } m \leqslant 10, \quad \rho=1 \quad \text { for } m>10
$$

our method could get the solution stably. The wave-forms are shown in Figures 5 and 6, where we assumed 128 frequency components. Wave-form (a) in Figure 5 is the response of the non-linear transmission line, while wave-form (b) shows the linear response neglecting non-linear terms. The features of the shock wave are apparent in the non-linear response. Wave-form (c) is the input impulse wave-form. The residual error defined by (12) for $M^{\prime}=256$ is given by

$$
\Delta_{128}=0.97 \times 10^{-2}
$$

The error in the amplitude is about $2 \cdot 4 \%$, which seems to be accurate enough.

We remark that although the perturbation method never converges for the stronger non-linear term

$$
v_{\mathrm{C}}=10^{11} q_{\mathrm{C}}+0.8 \times 10^{34} q_{\mathrm{C}}^{3}
$$

with $\Delta=0$, our perturbation method with $\Delta=0 \cdot 1$ can get the solution stably.

Thus we found from the example that our perturbation method is greatly improved by incorporating the two techniques of compensation and homotopy. 


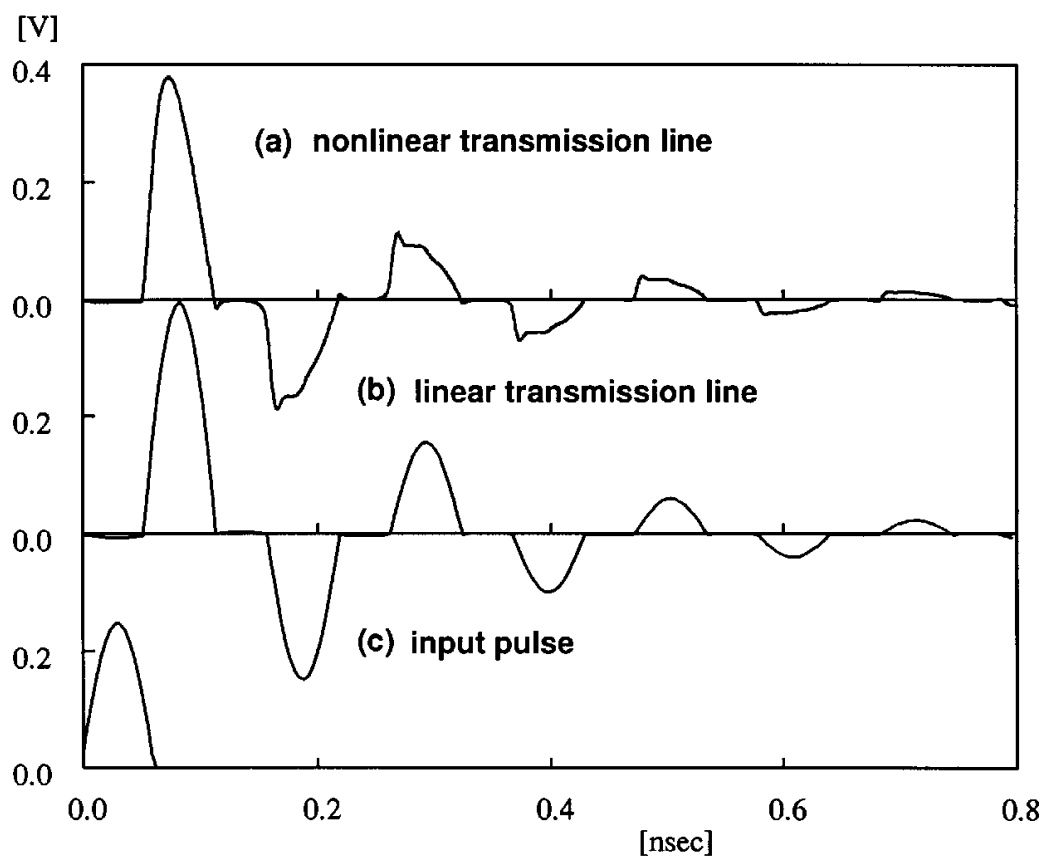

Fig. 5. Impulse responses: (a) response of non-linear transmission line; (b) response of linear transmission line neglecting non-linear terms; (c) input impulse, $\left(E_{\mathrm{m}}=0 \cdot 25 V, T=1 \mathrm{~ns}\right.$, and $k=16$ in (3))

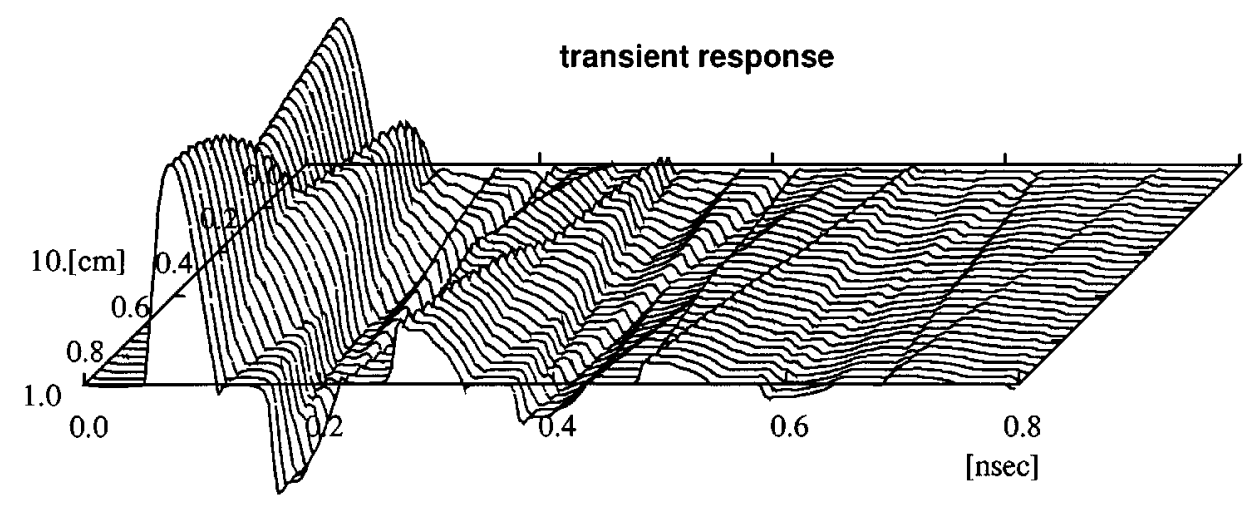

Fig. 6. Response of non-linear transmission line in $(x, t)$ domain $\left(l=10 \mathrm{~cm}, T=1 \mathrm{~ns}, E_{\mathrm{m}}=0 \cdot 25 \mathrm{~V}, R_{\mathrm{L}}=10 \Omega\right)$

\section{CONCLUSIONS AND REMARKS}

We have presented a frequency domain perturbation algorithm for calculating transient responses of non-linear transmission lines. We can greatly improve the convergence by introducing two techniques, the compensation method and the homotopy method. They have the properties of improving the convergence ratios and stabilizing the iteration by reducing the perturbed terms. Thus our perturbation method becomes much more powerful for the analysis of non-linear transmission lines.

Note that since the algorithm in this paper is based on the perturbation method, convergence may not be guaranteed even if we choose any $\Delta$ and $\Delta \rho$ for strong non-linearity. The non-linearity of the element depends on the amplitude of the input variable and can be estimated by the ratio (non-linear term)/ 
(linear term). In the example of (2) we have about $0 \cdot 5$ at $v_{\mathrm{C}}=0.4$. We found from our numerical experiences that we cannot hope for convergence when the ratio is greater than unity.

Most practical circuits such as GaAs transmission lines ${ }^{1}$ belong to the class of non-linear $R C$ transmission lines. They are special cases of our example where our algorithm can be efficiently applied to much stronger non-linearity.

\section{REFERENCES}

1. M. J. W. Rodwell, 'Nonlinear transmission line for picosecond pulse compression and broadband phase modulation', Electron. Lett., 23, 109-110 (1987).

2. R. K. Arora and J. L. Thaker, 'Electromagnetic pulse transmission on superconducting interconnects', Int. J. Electron., 71, 117-125 (1991).

3. C. J. Madden, R. A. Marsland, M. J. W. Rodwell, D. M. Bloom and Y. C. Pao, 'Hyperabrupt-doped GaAs nonlinear transmission line for picosecond shock wave generation', Appl. Phys. Lett., 54, 1019-1021 (1989).

4. K. S. Champlin and D. R. Singh, 'Small-signal second-harmonic generation by a non-linear transmission line', IEEE Trans. Microwave Theory Tech., MTT-34, 351-353 (1986).

5. C. Camacho-Penalosa and C. S. Aitchison, 'Analysis and design of MESFET gate mixers', IEEE Trans. Microwave Theory Tech., MTT-35, 643-652 (1987).

6. F. A. Benson and J. D. Last, 'Non-linear-transmission-line harmonic generator', Proc. IEE, 112, 635-643 (1965).

7. R. Landauer, 'Shock waves in nonlinear transmission lines and their effect on parametric amplification', IBM J., October, pp. 391-401 (1960).

8. D. Jager and F. -J. Tegude, 'Non-linear wave propagation along periodic-loaded transmission line', Appl. Phys., 15, $393-397$ (1978).

9. C. Camacho-Penalosa and I. Molina-Fernandez, 'Harmonic balance analysis of non-linear transmission lines', Electron. Lett., 24, 1235-1236 (1988).

10. R. H. Freeman and A. E. Karbowiak, 'An investigation of nonlinear transmission lines and shock waves', J. Phys. D: Appl. Phys., 10, 633-643 (1977)

11. I. Molina-Fernandez, C. Camacho-Penalosa and J. Ramos, 'Application of the two-dimensional Fourier transform to non linear wave propagation phenomena', IEEE Trans. Microwave Theory Tech., 42, 1079-1085 (1994).

12. C. A. Marinov and P. Peittaanmaki, 'A delay time bound for distributed parameter circuits with bipolar transistors', Int. j. cir. theor. appl., 18, 99-106 (1990).

13. J. L. Wyatt Jr., 'Monoton sensitivity of nonlinear nonuniform RC transmission lines with application to timing analysis of digital MOS integrated circuits', IEEE Trans. Circuits and Systems, CAS-32, 28-83 (1985).

14. C. B. Garcia and W. I. Zangwill, Pathways to Solutions, Fixed Points, and Equilibria, Prentice-Hall, Englewood Cliffs, NJ, 1981.

15. S. M. Roberts and J. S. Shipman, Two-Point Boundary Value Problems: Shooting Methods, American Elsevier, New York, 1972.

16. J. Kevorkian and J. D. Cole, Perturbation Methods in Applied Mathematics, Springer, New York, 1981.

17. L. A. Zadeh and C. A. Desoer, Linear System Theory: the State Space Approach, McGraw-Hill, New York, 1963.

18. L. O. Chua and P.-M. Lin, Computer-Aided Analysis of Electrical Circuits: Algorithms and Computational Techniques, PrenticeHall, Englewood Cliffs, NJ, 1975.

19. M. Urabe, 'Galerkin's procedure for nonlinear periodic systems', Arch. Rat. Mech. Anal., 20, 120-152 (1965).

20. A. Ushida, T. Adachi and L. O. Chua, 'Steady-state analysis of non-linear circuits based on hybrid method', IEEE Trans. Circuits and Systems-I, CAS 39, 649-661 (1992). 